\title{
Study on the Binder Distribution related to Drying
}

\author{
Ying $\mathrm{Li}^{1, \mathrm{a}}$, Qinming Wang ${ }^{1}$, Wenjuan $\mathrm{Gu}^{1}$ and Banggui $\mathrm{He}^{1}$ \\ ${ }^{1}$ Faculty of Mechanical and Electrical Engineering, Kunming University \\ of Science and Technology, Kunming, 650093, China \\ Email:haishanying@126.com
}

\begin{abstract}
.
The main aim of this study was to obtain the relationship between drying method and binder distribution in the z-direction. The results implied that it was reliable and effective method using LSCM to characterize the $\mathrm{z}$ - directional distribution of binder and analyze the influence of dry method on binder migration. The results indicated that the dry method was critical to binder distribution in coated paper and vacuum drying accelerated binder migration to the base paper. Infrared drying was good to producing little binder migration towards the interior of base paper, but infrared drying was disadvantageous to even binder distribution. Based on the results presented in this study, infrared drying seemed to be less desirable because it produced more uneven binder distributions that could potentially result in larger printing defects. Vacuum drying resulted in more even changes of binder distribution, which would produce better print smoothness.
\end{abstract}

Keywords: Drying Method; Distribution depth; Distribution Evenness; SEM; LSCM

\section{Introduction}

The coating structure and surface topography are dependent on factors such as pore structure, coating color formulation, particle arranging, coating speed and drying temperature, et. The amount and type of latex binder, pigment particle size, and coating condition have a significant effect on both the structure and chemical properties of pigment coatings, which affects critical surface properties, e.g., gloss, ink setting rate via liquid absorption, and spreading properties [1-4]. 
Some researchers have studied the binder distribution in recent years [5, 6]. In this study, the main aim was to characterize the z-directional distribution of the binder and obtain the influence of drying method on the binder distribution. Rhodamine B was used to stain the binder and as the probe to characterize z-directional distribution of the binder using LSCM. This method developed can be applied to analyze the degree of binder migration in coated papers.

\section{Experimental}

Preparations of the Coating Samples. The basis weight of uncoated base paper (supplied by Dong Tang Paper Mill) was $70 \mathrm{~g} / \mathrm{m}^{2}$.The coatings of paper were composed with kaolin pigment (supplied by Mao Ming Clay Company, China) and carboxylic styrene-butadiene latex (supplied by BASF Company, China). Rhodamine B (Sigma-R6626, Co. Ltd) was used as fluorescent dye for staining with the binder because it was successfully applied in some studies [7, 8]. Rhodamine B was dissolved in ethanol to reach $0.05 \mathrm{wt} \%$. $0.05 \mathrm{wt} \%$ of Rhodamine B was added to carboxylic styrene-butadiene latex, and then it was stirred. The binder was held to stain completely for $24 \mathrm{~h}$ before coating in order to make Rhodamine B and binder become the whole. The coating components are indicated in Table 1. Coating was performed with a bar coater (model K303 Multi-coater, RK Print Coat Instruments Ltd, United Kingdom).

- Coating speed $=4 \mathrm{~m} / \mathrm{min}$

- Drying temperature $=110^{\circ} \mathrm{C}$

- Drying time $=1 \mathrm{~min}$

- Coating thickness $=4 \mu \mathrm{m}$

Measurements of Scanning Electron Microscopy. Scanning electron microscopy (Hitachi S3700N, Japan) was utilized to investigate the elemental composition of the coatings surface. For SEM observation, all coating samples were gold coated and 20KV beam energy was used to get the information of elemental composition of the coatings. 
Table 1 Coating color recipes

\begin{tabular}{cccccc}
\hline Sample & Kaolin $\left(\mathrm{pph}^{*}\right)$ & $\mathrm{SB}\left(\mathrm{pph}^{*}\right)$ & Solid $(\%)$ & Coating thickness $(\mu \mathrm{m})$ & Drying Method \\
\hline F1 & 100 & 15 & 60 & 4 & Vacuum drying \\
F2 & 100 & 15 & 60 & 4 & Hot air drying \\
F3 & 100 & 15 & 60 & 4 & Infrared drying \\
\hline
\end{tabular}

\section{Parts per hundred}

Laser Scanning Confocal Microscopy. A Laser Scanning Confocal Microscopy (Leica TCS-SP5) was used to obtain the information about the z-directional distribution of the binder. Immersion liquid was supplied by Leica. As the laser beam scanned the coating layer, the emitted fluorescent light was detected by a photo detector. Confocal beam splitter filter (DD458/514) was used to separate the fluorescent light. An excitation wavelength of 514nm was used and the detected wavelength ranged from $520 \mathrm{~nm}$ to $615 \mathrm{~nm}$. The second PMT was used to receive Argon laser signal. The pinhole size was $67.95 \mu \mathrm{m}$ and digital zoom was 1. Adjusting z-direction position was to obtain appropriate focus plane. Meanwhile, adjusting PMT gain and PMT offset was to get the best image. A $385.7 \times 385.7 \mu \mathrm{m}^{2}$ square was scanned for a period of about 2-10 minutes which decided by the depth of binder distribution in coated paper, speed and pixel format of scanning. The data were collected in 1024x1024 pixel format and $400 \mathrm{~Hz}$ speed. Confocal images were obtained with the XYZ scan mode and to characterize $\mathrm{XY}$ images in the $\mathrm{z}$ (thickness)-direction at intervals of $0.1 \mu \mathrm{m}$. Coating layer was cut to a sequence of optical images in the z-direction.

Table 2 SEM surface elemental composition in at.\% of coating surface

\begin{tabular}{cccc}
\hline & F1 & F2 & F3 \\
\hline $\mathrm{C}$ & 7.71 & 6.14 & 4.68 \\
$\mathrm{O}$ & 72.22 & 69.36 & 71.60 \\
$\mathrm{Si}$ & 9.80 & 11.77 & 11.62 \\
$\mathrm{Al}$ & 10.16 & 12.53 & 11.88 \\
$\mathrm{~K}$ & 0.11 & 0.20 & 0.22 \\
& & & \\
\hline
\end{tabular}




\section{Results and Discussion}

Study on the Binder Content of Coating Surface by SEM. Scanning electrical microscopy (SEM) was used to investigate the surface elemental composition of the coating samples. The surface composition in atomic percentage was calculated, and then was presented as energy spectrum. Results are shown in Tables 2. The Al, Si and part of the O signals come from the clay. The main part of the $\mathrm{C}$ signal originates from the SB latex. The amount of carbon originating from each one was calculated with the help of SEM software (Table 2). Table 2 shows the variation of surface composition with different drying method as found from SEM. In all coating samples investigated, the overall binder content is the same, i.e. binder content of coating surface differentiate with different drying method. Indeed, defects in printability observed after different drying conditions (e.g. print mottle) are often due to phenomena such as binder migration towards the coating surface. It can be concluded from Table 2 that Vacuum drying results in the highest amount of carbon content on the coating surface and Infrared drying results in the lowest amount of carbon content on the coating surface, which means that different drying method produces different binder migration towards the coating surface.

\section{Investigation into the Influence of Drying Method on Binder Distribution}

by LSCM. Binder was stained with Rhodamine B, so the fluorescent signals detected by LSCM represented the binder exiting. The fluorescent intensity of every image by LSCM represents the amount of the binder in this XY plane image. When laser scanned the coating surface, variation of the fluorescent intensity showed the variation of the amount of the binder in the z-direction. The profiles of binder z-directional distribution were obtained by calculating fluorescent intensity of all XY plane images using Leica software. Fig.1 displays the fluorescent intensity distribution in the z-direction (thickness) and illustrates the $z$-directional binder profiles after image processing and analysis using leica software. It could be summarized the effect of drying method on the binder content and uniformity in the z-direction as measured by LSCM from Fig.1. 

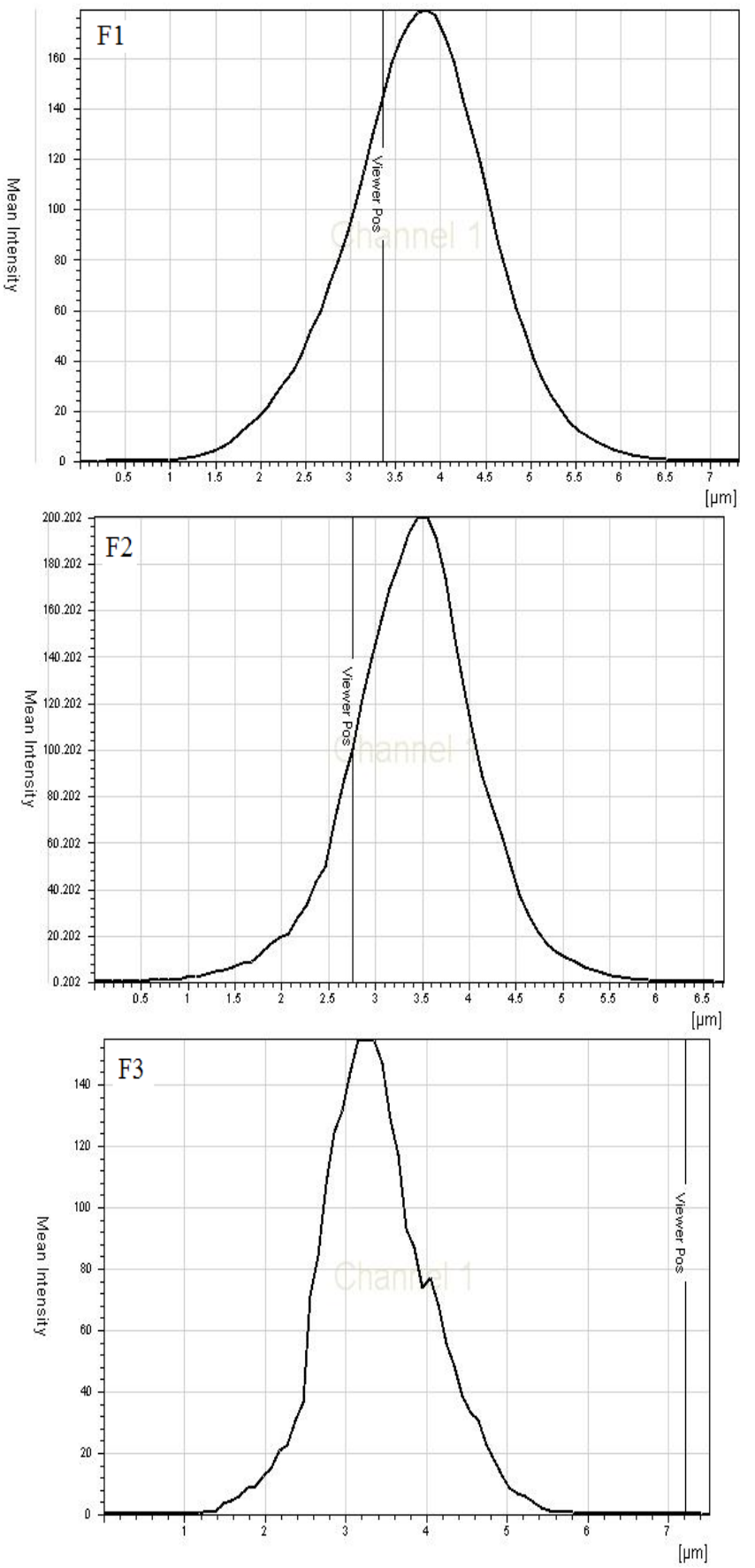

Fig.1. Profiles of the binder z-directional distribution of coating samples which dried with different drying methods. 
The fluorescent intensity distribution maps (Fig.2) illustrated binder distribution in the z-direction from numerical characterization. Sample F3 had a quite narrow distribution mainly ranging from $1.4 \mu \mathrm{m}$ to $5.6 \mu \mathrm{m}$, which implied that sample F3 had the narrowest binder distribution in these samples. It was clear shown in Fig.2 that the depth distribution of sample F1 ranging between $0.5 \mu \mathrm{m}$ and $6.5 \mu \mathrm{m}$ and the depth distribution of sample F2 ranging between $0.6 \mu \mathrm{m}$ and $5.8 \mu \mathrm{m}$. It showed that sample F1 had a broadest binder distribution in these samples. The results indicated that the samples differed in fluorescent intensity distribution in z-direction, so the binder z-directional distribution was different due to different drying method. The z-direction binder profiles suggested that there was a correlation between drying method and binder distribution. The correlation was observed from the two aspects that describe uniformity: symmetry of profile changing and smooth degree of the profile for the z-directional binder distribution. The coating thickness of overall samples was $4 \mu \mathrm{m}$, so the binder must penetrate into the base paper when $\mathrm{z}$-direction length detected by LSCM was larger than $4 \mu \mathrm{m}$. The samples F1 yielded a higher depth value of binder z-directional penetration into base paper compared with the other samples. The z-direction length of sample F1 is the largest, which indicate that it is the highest degree of binder migration to the base paper in coating sample F1. There was little binder migration towards base paper in sample F3.It could be concluded that vacuum drying resulted in higher amount of the binder penetration into base paper. This result was a consequence of that speed of heat penetrating into paper interior slowed and drying speed of paper slowed, which made water have more time to carry binder to penetrate into the base paper (absorption). The degree of binder penetrating into base paper of sample F2 was larger than sample F3, which resulted from different method of heat transfer between hot air drying and infrared drying. The method of heat transfer of hot air drying was convection and the method of heat transfer of hot infrared drying was heat radiation. Infrared drying had very strong penetrability, so temperature of coating interior increased shortly. Increased temperature destroyed the hydrogen bond and reduced the ability of water transferring binder, so hot air drying 
accelerates water to carry binder migration to base paper compared with infrared drying.

\section{Conclusions}

The LSCM can be applied to analyze the degree of binder migration in coated papers. The z-directional distribution of binder was showed in the method of numerical characterization by LSCM. A coated paper binder profile with variations in the $\mathrm{z}$ - direction variations observed gave an indication of the presence of binder migration. It could be concluded that vacuum drying resulted in the highest amount of the binder penetration into base paper, coating surface and the most uniform distribution of binder compared with the other drying method. It is also found that infrared drying creates a more uneven binder arrangement in the z-direction but little binder migration to base paper. Vacuum drying was advantageous for binder even distribution and infrared drying be good to decreasing the degree of binder migration to the base paper. More unevenness in the binder distribution can create higher local variations in pore structure, and in turn, spatial variations in absorption that can yield mottling.

\section{Acknowledgements}

The authors express their gratitude to the Yunnan Province Science and Technology Department (Project KKSY 2012/01059 and KKSY 2012/01051) for financial support.

\section{References}

[1] J.S. Preston, N.J. Elton, A. Legrix and C. Nutbeem: Proceeding of TAPPI Advanced Coating Fundamentals Symposium, San Diego, May 2001.

[2] S. Rousu, P.A.C. Gane, D. Speilmann: Nordic Pulp Paper Res. J. 15 (5) (2001) p.527.

[2] Y. Xiang, D. Desjumaux, D. Bousfield and M.F. Forbes: Preprints of the Pan Pacific and International Printing and Graphic Arts Conference, Quebec, 
1998, p. 85.

[4] G. Ström, A. Englund, M. Karathanasis: Nordic Pulp and Paper Research J. 18(1) (2003) p.10

[5] C. Kugge: J. Pulp Paper Sci. 30 (4) (2004) p.105-111.

[6] R.N.S. Sodhi, L. Sun, M. Sain and R. Farnood: J. Adhesion 84 (3) (2008) p.277-292.

[7] Y. Ozaki, D.W. Bousfield and S.M. Shaler: Journal of pulp and paper science 31(1) (2005) p.48-52.

[8] Y. Ozaki, D.W. Bousfield and S.M. Shaler: TAPPI J. 5(2) (2006) p.3-8. 